\title{
Article
}

Mycosphere

\section{Phylogenetic placement of Micropeltidaceae}

\section{Hongsanan S and Hyde KD}

\author{
Center of Excellence in Fungal Research, Mae Fah Luang University, Chiang Rai 57100, Thailand
}

Hongsanan S, Hyde KD 2017 - Phylogenetic placement of Micropeltidaceae. Mycosphere 8(10), 1930-1942, Doi 10.5943/mycosphere/8/10/15

\begin{abstract}
"Microthyriaceae-like" taxa are fungal epiphytes which appear as black dots on the host surface. The families Micropeltidaceae and Microthyriaceae have been poorly studied, particularly with molecular data, due to the difficulty in obtaining pure cultures. The two families were placed in Microthyriales in many studies based on thyriothecial characters. Two species of Micropeltidaceae (Micropeltis dendrophthoes and M. zingiberacicola) clustered at the base of Dothideomycetes and were unrelated to Microthyriaceae in previous phylogenetic trees. Their placements were treated as unresolved. We restudied sequence data of Micropeltidaceae and its related strains to clarify the current placement using authentic strains available in GenBank. Phylogenetic analyses generated from maximum likelihood and Bayesian analyses (used ITS, LSU, RPB2, SSU and TEF1 sequence data) including Blast results, indicate that the current placement of Micropeltidaceae is in Ostropales (Lecanoromycetes), although it is not strongly supported. A revised phylogenetic tree for Micropeltidaceae and selected families from Dothideomycetes, Eurotiomycetes, Lecanoromycetes, Leotiomycetes and Sordariomycetes is provided with discussions in this paper.
\end{abstract}

Key words - Dothideomycetes- Lecanoromycetes- Microthyriales - Phylogeny

\section{Introduction}

The family Micropeltidaceae was introduced by Clements \& Shear in 1931 as 'Micropeltaceae'. The family represents foliar, biotrophic epiphytes, which mostly can be found on the lower leaf surface as small black dots. Species in this family are characterized by flattened, black-blue or greenish to black thyriothecia, easily removed from the host surface, poorly developed at the base, comprising interwoven hyphae, with a central ostiole, and up to multi transseptate, hyaline ascospores (Clements \& Shear 1931, Batista 1959, von Arx \& Müller 1975, Barr 1987, Kirk et al. 2008, Lumbsch \& Huhndorf 2010, Wu et al. 2011, Hyde et al. 2013, Hongsanan et al. 2015a). Micropeltidaceae and Microthyriaceae were placed in Microthyriales (Dothideomycetes) based on their flattened, easily removed thyriothecia, and poorly-developed base. However, thyriothecium of Microthyriaceae comprise cuboid or angular cells, arranged in parallel rows from the prominent central ostiole and uniseptate ascospores (Doidge 1942, Müller \& von Arx 1962, Luttrell 1973, Barr 1987, Hofmann \& Piepenbring 2006, Hofmann 2010, Wu et al. 2011, Hyde et al. 2013, Hongsanan et al. 2015a).

Most of species with Microthyrium-like characters cannot be cultured; therefore, little molecular data is available until now. Schoch et al. (2009) provided a single strain of Microthyrium microscopicum (type species of Microthyriaceae) which represented the order Microthyriales as a 
long branch sister to Stomiopeltis betulae (considered as Micropeltidaceae) in phylogenetic trees (Wu et al. 2011, Hyde et al. 2013). Micropeltis zingiberacicola, Neomicrothyrium siamense, Paramicrothyrium chinensis and Stomiopeltis versicolor were introduced to accommodate as Microthyriaceae-like taxa, but they did not cluster with Microthyrium microscopicum in the phylogenetic tree (Wu et al. 2011). Therefore, the placement of Microthyriaceae was not confirmed, while the placement of Micropeltidaceae was unresolved (Wu et al. 2011). Hongsanan et al. (2014a) and Ariyawansa et al. (2015) added a new genus and species in Microthyriaceae based on morphology and molecular data; this supported the status of Microthyriaceae in Dothideomycetes. Although, Hongsanan et al. (2015a) established a new species in Micropeltidaceae with sequence data, their phylogenetic tree only distinguished the new species. Presently, there are only two species of Micropeltidaceae that were established with sequence data, thus the placement of Micropeltidaceae has not been well resolved until now.

In this study, we use sequence data from previous studies to restudy the placement of Micropeltidaceae by Blast searches and phylogenetic analysis of combined ITS, LSU, RPB2, SSU, and TEF1 sequence data. The placement of this family is presented in the phylogenetic tree (Figs 1, 2 ), and the discussion of the new placement is provided.

\section{Materials \& Methods}

\section{Phylogenetic analyses}

Sequence data of Micropeltidaceae from previous studies were downloaded from GenBank to supplement the dataset (Table 1), and representative strains of major classes in Ascomycota were also downloaded. The data sets were aligned for each gene partition using MAFFT (Katoh et al. 2009), and checked manually using Bioedit (Hall 1999). Schizosaccharomyces pombe was selected as the outgroup taxon. Maximum likelihood analysis was performed by using raxmlGUIv. $0.9 \mathrm{~b} 2$ for each gene partitioned and combined dataset (Silvestro \& Michalak 2012), using GTRGAMMA model of nucleotide substitution, and the search strategy was set to rapid boot strapping. Maximum likelihood bootstrap values equal or greater than $70 \%$ are shown as the first set of numbers at the nodes (Fig. 1). The best fit model of evolution was performed by MrModeltest 2.2 (Nylander et al. 2008). Posterior probabilities (PP) (Rannala \& Yang 1996, Zhaxybayeva \& Gogarten 2002) were performed in MrBayes v 3.1.2 by Markov Chain Monte Carlo sampling (MCMC) (Huelsenbeck \& Ronquist 2001). The six simultaneous Markov chains were run for $1,000,000$ generations, with sampling frequency at 100 . The total 10,000 trees were obtained; however, the first 2,000 trees were discarded based on the suggestion from Tracer software. The remaining 8,000 trees were calculated for posterior probabilities (Cai et al. 2006, 2008). The posterior probabilities (PP) equal or greater than 0.90 are given as the second set of numbers at the nodes (Figs 1,2).

\section{Phylogenetic analysis}

The combined analyses of LSU, RPB2, SSU and TEF1 sequence data from representative strains of Dothideomycetes, Eurotiomycetes, Geoglossomycetes, Lecanoromycetes, Leotiomycetes and Orbiliomycetes (Fig. 1) and combined analyses of ITS, LSU, SSU and TEF1 sequence data from Stictidaceae and related families (Fig. 2) were used in this study. The topology of each gene partition is mostly similar to the combined data set, except some unstable strains. Lecanoromycetes Clade includes representative strains of families in Lecanoromycetes. Micropeltis zingiberacicola is closely related to $M$. dendrophthoes (100\% ML and 1.0 PP), they cluster in Ostropales within Lecanoromycetes and closely related to Cyanodermella asteris strain 03HOR06-2-4 and Cyanodermella oleoligni strain DTO 301-G1 (100\% ML and 1.0 PP). In the Dothideomycetes clade, Microthyriaceae represented by Microthyrium microscopicum and M. buxicola clusters as a sister group to the clade containing strains from Natipusillales, Venturiales and Zeloasperisporiales $(0.9 \% \mathrm{PP})$. 
Table 1 Taxa used in the phylogenetic analysis and GenBank accession numbers (ITS, LSU, RPB2, SSU and TEF1) and species voucher/culture numbers.

\begin{tabular}{|c|c|c|c|c|c|c|}
\hline \multirow{2}{*}{ Species } & \multirow{2}{*}{ Voucher/culture } & \multicolumn{5}{|c|}{ Accession numbers } \\
\hline & & LSU & SSU & ITS & $R P B 2$ & TEF1 \\
\hline Acarospora laqueata & AFTOL-ID 1007 & AY640943 & AY640984 & DQ842014 & - & - \\
\hline Acarosporina microspora & AFTOL-ID 78 & AY584643 & AY584667 & DQ782834 & AY584682 & DQ782890 \\
\hline Agonimia sp. & AFTOL 684 & DQ782913 & DQ782885 & DQ826742 & DQ782874 & DQ782917 \\
\hline Agyrium rufum & Wedin 7931 & EF581826 & EF581823 & JX000097 & - & - \\
\hline Agyrium rufum & Buck 48698 & EF581825 & EF581824 & - & - & - \\
\hline Aigialus grandis & JK 5244A & GU301793 & GU296131 & - & GU371762 & - \\
\hline Anzia colpodes & Lumbsch 4.VI.04 & DQ923651 & DQ923622 & - & - & - \\
\hline Apiospora montagnei & AFTOL-ID 951 & DQ471018 & - & - & DQ470921 & DQ842027 \\
\hline Arctomia delicatula & P166 & KR017191 & KR017335 & - & - & KR017563 \\
\hline Arthrorhaphis alpina & Spribille 26526 & KP794950 & - & - & - & - \\
\hline Arthrorhaphis citrinella & AFTOL-ID 2341 & AY853356 & - & - & - & - \\
\hline Aspergillus fumigatus & ATCC 1022 & AY660917 & AB008401 & KF314726 & - & - \\
\hline Aspergillus nidulans & ATCC 16855 & AF454167 & ENU77377 & - & - & - \\
\hline Asterina melastomatis & VIC 42822 & KP143739 & - & - & - & - \\
\hline Aureobasidium pullulans & CBS 584.75 & FJ150942 & EU682922 & NR_144909 & KT693976 & FJ157895 \\
\hline Australiasca queenslandica & BRIP 24607 & HM237324 & - & HM237327 & - & - \\
\hline Baeomyces rufus & P78 & KJ462341 & KR017260 & KJ462264 & - & KR017567 \\
\hline Bombardia bombarda & AFTOL-ID 967 & DQ470970 & DQ471021 & - & DQ470923 & DQ471095 \\
\hline Botryosphaeria dothidea & AFTOL-ID 946 & DQ678051 & FJ190612 & - & DQ677944 & DQ767637 \\
\hline Byssoloma subdiscordans & AFTOL-ID 4884 & KJ766538 & KJ766696 & - & - & - \\
\hline Calicium salicium & CBS 100898 & KF157982 & KF157970 & - & KF157998 & \\
\hline Calicium viride & 10-VII-1997 (DUKE) & AF356670 & AF356669 & - & - & - \\
\hline Calycidium cuneatum & Wedin $8034(\mathrm{~S})$ & JX000083 & - & JX000114 & - & - \\
\hline Candelaria concolor & AFTOL-ID 1706 & DQ986791 & DQ986806 & - & - & - \\
\hline Candelariella reflexa & AFTOL-ID 1271 & DQ912331 & DQ912309 & - & DQ912380 & - \\
\hline Canoparmelia caroliniana & AFTOL-ID 6 & AY584634 & AY584658 & DQ782833 & - & DQ782889 \\
\hline Capnodium coartatum & MFLUCC10-0069 & JN832614 & JN832599 & - & - & - \\
\hline Capnodium coffeae & CBS 147.52 & DQ247800 & DQ247808 & AJ244239 & KT216519 & \\
\hline Capronia pilosella & AFTOL-ID 657 & DQ823099 & DQ823106 & DQ826737 & DQ840561 & DQ840565 \\
\hline
\end{tabular}


Table 1 Continued.

\begin{tabular}{|c|c|c|c|c|c|c|}
\hline \multirow{2}{*}{ Species } & \multirow{2}{*}{ Voucher/culture } & \multicolumn{5}{|c|}{ Accession numbers } \\
\hline & & LSU & SSU & ITS & $R P B 2$ & TEF1 \\
\hline Carestiella social & Gilenstam 2437a & AY661682 & - & AY661682 & - & - \\
\hline$c$ & MFLUCC 10-0008 & HQ895835 & - & NR_137768 & - & - \\
\hline Chaenothecopsis montana & HT435 & KF157987 & KF157975 & -- & KF158002 & - \\
\hline Chaenothecopsis sitchensis & HT22 & KF157988 & KF157976 & - & KF158003 & - \\
\hline Chaetomidium galaicum & CBS 113678 & FJ666361 & - & - & FJ666392 & - \\
\hline Chaetosphaerella fusca & GKML124N & FJ968967 & - & - & - & FJ969002 \\
\hline Chaetosphaeria innumera & SMH 2748 & AY017375 & - & - & - & - \\
\hline Chlorociboria aeruginosa & AFTOL-ID 151 & AY544669 & AY544713 & DQ491501 & DQ470886 & DQ471053 \\
\hline Cladophialophora carrionii & CBS 260.83 & KF928518 & AY554285 & KF928454 & - & EU137234 \\
\hline Clypeosphaeria uniseptata & HKUCC 6349 & DQ810219 & DQ810255 & - & - & - \\
\hline Coccomyces dentatus & AFTOL-ID 147 & AY544657 & AY544701 & DQ491499 & DQ247789 & DQ497605 \\
\hline Coccomycetella richardsonii & Baloch SW068 & HM244761 & HM244737 & - & HM244785 & - \\
\hline Coccotrema cucurbitula & - & AF274092 & AF274114 & AF329162 & - & - \\
\hline Coccotrema maritimum & - & AF329164 & AF329163 & AF329165 & - & - \\
\hline Coccotrema pocillarium & - & AF274093 & AF274113 & AF329167 & - & - \\
\hline Cochliobolus heterostrophus & AFTOL-ID 54 & AY544645 & AY544727 & DQ491489 & DQ247790 & DQ497603 \\
\hline Coenogonium disjunctum & - & AF465443 & AF465458 & - & - & - \\
\hline Coenogonium leprieurii & - & AF465442 & AF465457 & - & - & - \\
\hline Colletotrichum gloeosporioides & LC0555 & JN940412 & JN940356 & JN943090 & - & - \\
\hline Conidiocarpus caucasicus & GUMH937 & KC833050 & KC833051 & - & - & - \\
\hline Coniocessia nodulisporioides & Co108 & GU553349 & - & GU553338 & - & - \\
\hline Crocynia pyxinoides & AFTOL-ID 111 & AY584653 & AY584677 & - & DQ883748 & DQ883767 \\
\hline Cryptadelphia groenendalensis & SH12 & EU528007 & - & - & - & - \\
\hline Cryptodiscus cladoniicola & RP159 & KY661652 & KY661694 & KY661619 & - & - \\
\hline Cryptodiscus foveolaris & EB88 & FJ904671 & - & - & - & - \\
\hline Cryptodiscus gloeocapsa & Vezda 1198 & AF465440 & AF465456 & - & - & - \\
\hline Cryptodiscus tabularum & EB87 & FJ904688 & - & FJ904688 & - & - \\
\hline Curvularia brachyspora & AFTOL-ID 354 & AF279380 & L36995 & - & - & - \\
\hline Curvularia fallax & FSU $<$ DEU $>: 5293$ & KF157989 & KF157977 & - & KF158004 & - \\
\hline Cyanodermella asteris & 03HOR06-2-4 & KT758843 & - & KT758843 & KU934214 & - \\
\hline
\end{tabular}


Table 1 Continued.

\begin{tabular}{|c|c|c|c|c|c|c|}
\hline \multirow{2}{*}{ Species } & \multirow{2}{*}{ Voucher/culture } & \multicolumn{5}{|c|}{ Accession numbers } \\
\hline & & LSU & SSU & $I T S$ & $R P B 2$ & TEF1 \\
\hline Cyanodermella oleoligni & DTO 301-G1 & KX950461 & - & KX950434 & KX999147 & - \\
\hline Cyphelium inquinans & Tibell 22283 & AY453639 & U86695 & AY450584 & - & - \\
\hline Cyphelium tigillare & Tibell 22343 & AY453641 & AF241545 & AY452497 & - & - \\
\hline Cyphellophora reptans & CBS 120903 & KF928542 & KF155209 & KF928478 & - & - \\
\hline Cyphellophora sessilis & CBS 238.93 & KF928523 & KC455309 & KF928459 & KC455289 & - \\
\hline Dermatocarpon miniatum & AFTOL-ID 91 & AY584644 & AY584668 & DQ782837 & DQ782863 & DQ782893 \\
\hline Dermea acerina & CBS 161.38 & DQ247801 & DQ247809 & - & - & - \\
\hline Diaporthe eres & AFTOL-ID 935 & AF408350 & DQ471015 & DQ491514 & DQ470919 & DQ479931 \\
\hline Dissoconium aciculare & CBS 342.82 & EU019266 & GU214524 & NR_119427 & - & - \\
\hline Dothidea sambuci & AFTOL-ID 274 & AY544681 & AY544722 & DQ491505 & DQ522854 & DQ497606 \\
\hline Elsinoe centrolobi & CBS 222.50 & DQ678094 & DQ678041 & KX887206 & KX887089 & KX886852 \\
\hline Elsinoe phaseoli & CBS 165.31 & DQ678095 & DQ678042 & KX887263 & KX887144 & KX886908 \\
\hline Exophiala dermatitidis & AFTOL-ID 668 & DQ823100 & DQ823107 & DQ826738 & DQ840562 & DQ840566 \\
\hline Glyphopeltis ligustica & Brusse 4947 & AY756337 & AY756399 & - & - & - \\
\hline Gnomonia gnomon & CBS 199.53 & AF408361 & DQ471019 & AY818956 & - & EU221885 \\
\hline Gregorella humida & Pykala 23630 & EU360846 & EU360867 & - & - & - \\
\hline Harknessia eucalypti & CPC 13643 & JQ706215 & - & JQ706089 & - & - \\
\hline Hymenelia epulotica & AFTOL-ID 1844 & KJ766569 & KJ766404 & - & KJ766975 & - \\
\hline Hypocrea americana & AFTOL-ID 52 & AY544649 & - & DQ491488 & - & DQ471043 \\
\hline Hysterium angustatum & CBS 236.34 & FJ161180 & GU397359 & - & FJ161117 & FJ161096 \\
\hline Hysterobrevium smilacis & CBS: 114601 & FJ161174 & FJ161135 & - & FJ161114 & FJ161091 \\
\hline Icmadophila ericetorum & AFTOL-ID 4846 & KJ766573 & KJ766729 & - & - & - \\
\hline Ingvariella bispora & BCNLich 17183 & HQ659185 & - & - & - & - \\
\hline Jahnula aquatica & R68-1 & EF175655 & EF175633 & JN942354 & - & - \\
\hline Jattaea mookgoponga & STE-U 6184 & EU367458 & EU367463 & EU367449 & - & - \\
\hline Kellermania yuccigena & CBS 131727 & KF766356 & KF766466 & KF766186 & - & KF766417 \\
\hline Lachnum virgineum & AFTOL-ID 49 & AY544646 & AY544688 & DQ491485 & DQ470877 & DQ497602 \\
\hline Lembosia abaxialis & VIC 42825 & KP143737 & - & - & - & - \\
\hline Leotia lubrica & AFTOL-ID 1 & AY544644 & AY544687 & DQ491484 & DQ470876 & DQ471041 \\
\hline Leptosphaerulina australis & CBS 317.83 & EU754166 & EU754067 & GU237829 & - & GU349070 \\
\hline
\end{tabular}


Table 1 Continued.

\begin{tabular}{|c|c|c|c|c|c|c|}
\hline \multirow{2}{*}{ Species } & \multirow{2}{*}{ Voucher/culture } & \multicolumn{5}{|c|}{ Accession numbers } \\
\hline & & LSU & SSU & ITS & $R P B 2$ & TEF1 \\
\hline Leptoxyphium cacuminum & MFLUCC10-0049 & JN832602 & JN832587 & - & - & - \\
\hline Lichenoconium aeruginosum & CBS 129239 & HQ174269 & HQ174268 & - & - & - \\
\hline Lichenoconium erodens & JL363-09 & HQ174267 & HQ174266 & - & - & - \\
\hline Lobothallia radiosa & AFTOL-ID 1860 & KJ766596 & KJ766746 & - & KJ766935 & - \\
\hline Loxospora cismonica & AFTOL-ID 878 & DQ986749 & DQ986742 & HQ650640 & DQ992433 & - \\
\hline Loxosporopsis corallifera & T1087 & KR017219 & KR017264 & KR017089 & KR017516 & KR017572 \\
\hline Lulworthia fucicola & ATCC 64288 & AY878965 & AY879007 & - & - & \\
\hline Magnaporthe salvinii & M21 & JF414887 & JF414862 & JF414838 & - & JF710406 \\
\hline Manglicola guatemalensis & BCC20157 & FJ743450 & FJ743444 & JN819282 & - & - \\
\hline Massaria inquinans & CBS 122369 & GU456322 & GU456300 & - & - & GU456282 \\
\hline Megalaria grossa & AFTOL-ID 4916 & KJ766597 & KJ766747 & - & - & - \\
\hline Megaspora verrucosa & L009 & HM060725 & HM060687 & - & - & - \\
\hline Microascus trigonosporus & AFTOL-ID 914 & DQ470958 & DQ471006 & - & - & - \\
\hline Micropeltis dendrophthoes & MFLUCC:15-0599 & KT588595 & KT588597 & - & - & - \\
\hline Micropeltis zingiberacicola & IFRDCC 2264 & JQ036227 & JQ036222 & - & - & - \\
\hline Microthyrium buxicola & MFLUCC 15-0213 & KT306552 & KT306550 & - & - & - \\
\hline Microthyrium microscopicum & CBS 115976 & GU301846 & GU296175 & - & - & GU349042 \\
\hline Miltidea ceroplasta & - & HQ391558 & HQ391557 & - & - & - \\
\hline Mollisia cinerea & AFTOL-ID 76 & DQ470942 & DQ470990 & DQ491498 & DQ470883 & DQ471051 \\
\hline Monascus purpureus & AFTOL-ID 426 & DQ782908 & DQ782881 & DQ782847 & DQ782869 & - \\
\hline Montagnula opulenta & CBS 168.34 & NG_027581 & - & - & - & - \\
\hline Mycoblastus affinis & AFTOL-ID 1047 & KJ766601 & - & - & KJ766958 & - \\
\hline Mycoblastus sanguinarius & AFTOL-ID 4865 & KJ766602 & KJ766440 & - & - & - \\
\hline Mycocalicium polyporaeum & ZW-Geo60-Clark & AY789362 & AY789361 & AY789363 & - & - \\
\hline Myeloconis erumpens & MPN778 & KJ449338 & - & - & - & - \\
\hline Myeloconis fecunda & MPN759 & KJ449337 & - & - & - & - \\
\hline Myriangium duriaei & CBS 260.36 & NG_027579 & AF242266 & - & KT216528 & - \\
\hline Mytilinidion mytilinellum & CBS 303.34 & FJ161184 & FJ161144 & - & FJ161119 & FJ161100 \\
\hline Natipusilla decorospora & L_A236_1A & HM196369 & HM196376 & - & - & - \\
\hline Natipusilla naponensis & $\mathrm{AF} 217-1 \mathrm{a}$ & HM196371 & HM196378 & - & - & - \\
\hline
\end{tabular}


Table 1 Continued.

\begin{tabular}{|c|c|c|c|c|c|c|}
\hline \multirow{2}{*}{ Species } & \multirow{2}{*}{ Voucher/culture } & \multicolumn{5}{|c|}{ Accession numbers } \\
\hline & & LSU & SSU & ITS & $R P B 2$ & TEF1 \\
\hline Neofracchiaea callista & SMH2689 & AY695269 & - & - & FJ968941 & FJ969020 \\
\hline Neophyllis melacarpa & - & AY340556 & - & - & - & - \\
\hline Nitschkia tetraspora & GKML148N & FJ968987 & - & - & FJ968936 & FJ969011 \\
\hline Ochrolechia tartarea & DNA7 & JN941358 & - & JN943620 & - & - \\
\hline Odontotrema phacidiellum & Gilenstam 2625 & HM244769 & - & - & HM244802 & - \\
\hline Ophioceras commune & M92 & JX134688 & JX134662 & JX134676 & - & JX134702 \\
\hline Ophiocordyceps sinensis & YN09_64 & JX968033 & JX968028 & JQ325141 & JX968013 & JX968018 \\
\hline Ophiostoma piliferum & AFTOL-ID 910 & DQ470955 & DQ471003 & - & DQ470905 & DQ471074 \\
\hline Orbilia auricolor & AFTOL-ID 906 & DQ470953 & DQ471001 & DQ491512 & DQ470903 & DQ471072 \\
\hline Orbilia vinosa & AFTOL-ID 905 & DQ470952 & DQ471000 & DQ491511 & - & DQ471071 \\
\hline Orceolina kerguelensis & - & AF274116 & - & - & - & DQ366254 \\
\hline Paramicrothyrium chinensis & IFRDCC2258 & KF636760 & JQ036224 & KM246198 & - & - \\
\hline Parmelia saxatilis & Wedin 5051 & AY300849 & AF117985 & - & - & - \\
\hline Parmularia styracis & VIC 42447 & KP143728 & - & KP273230 & - & KP289325 \\
\hline Peltula auriculata & AFTOL-ID 892 & DQ832330 & DQ832332 & DQ832329 & DQ832331 & - \\
\hline Peltula umbilicata & AFTOL-ID 891 & DQ832334 & DQ782887 & DQ832333 & DQ832335 & DQ782919 \\
\hline Pertusaria dactylina & AFTOL-ID 224 & DQ782907 & DQ782880 & DQ782843 & DQ782868 & DQ782899 \\
\hline Phaeosaccardinula ficus & MFLUCC 10-0009 & HQ895837 & - & HQ895840 & - & KF791915 \\
\hline Phaeosphaeria oryzae & CBS 110110 & GQ387591 & GQ387530 & KF251186 & KF252193 & - \\
\hline Phlyctis argena & AFTOL-ID 1375 & DQ986771 & DQ986880 & - & KJ766940 & - \\
\hline Phyllobaeis imbricata & AFTOL-ID 852 & DQ986781 & DQ986895 & HQ650635 & - & - \\
\hline Physcia aipolia & AFTOL-ID 84 & DQ782904 & DQ782876 & DQ782836 & DQ782862 & DQ782892 \\
\hline Platystomum scabridisporum & BCC 22835 & GQ925844 & GQ925831 & - & GU479830 & GU479857 \\
\hline Plectosphaerella cucumerina & DAOM 226828 & GU180647 & GU180612 & GU180630 & GU180663 & - \\
\hline Pleospora herbarum & CBS 191.86 & DQ247804 & DQ247812 & KC584239 & KC584471 & KC584731 \\
\hline Pleurostomophora richardsiae & CBS H-7595 & AY761080 & AY761066 & - & - & - \\
\hline Porina farinosa & MPN36 & KJ449333 & KJ449312 & - & - & - \\
\hline Porina nucula & MPN13B & KJ449331 & KJ449310 & & - & - \\
\hline Potebniamyces pyri & AFTOL-ID 744 & DQ470949 & DQ470997 & DQ491510 & DQ470900 & DQ471068 \\
\hline Protothelenella corrosa & 2002 Palice & AY607734 & - & - & - & - \\
\hline
\end{tabular}


Table 1 Continued.

\begin{tabular}{|c|c|c|c|c|c|c|}
\hline \multirow{2}{*}{ Species } & \multirow{2}{*}{ Voucher/culture } & \multicolumn{5}{|c|}{ Accession numbers } \\
\hline & & LSU & SSU & ITS & $R P B 2$ & TEF1 \\
\hline Protothelenella sphinctrinoidella & Lumbsch 19031d (F) & AY607735 & AY607747 & - & - & - \\
\hline Pseudovalsa longipes & AR 3541 & EU683072 & - & - & - & - \\
\hline Psora decipiens & AFTOL-ID 4857 & KJ766640 & KJ766778 & - & - & - \\
\hline Pyrenula pseudobufonia & Reeb VR 14-VI-02/5 (DUKE) & AY640962 & AY641001 & - & - & - \\
\hline Pyrgillus javanicus & AFTOL-ID 342 & DQ823103 & DQ823110 & DQ826741 & DQ842009 & - \\
\hline Ramalina farinacea & AFTOL-ID 1965 & KJ766646 & KJ766783 & - & KJ766963 & - \\
\hline Ramichloridium anceps & AFTOL-ID 659 & DQ823102 & DQ823109 & DQ826740 & DQ840564 & DQ840568 \\
\hline Schaereria corticola & Toensberg 28432 & AY300859 & AY300909 & - & - & - \\
\hline Schizoparme straminea & CBS 149.22 & AF362569 & - & - & - & - \\
\hline Schizosaccharomyces pombe & Z19136 & X54866 & - & - & - & - \\
\hline Siphula ceratites & $\mathrm{P} 110$ & KR017179 & KR017288 & KR017095 & KR017498 & KR017625 \\
\hline Sordaria fimicola & HP153 & KT323354 & - & KT323211 & - & - \\
\hline Sphaerophorus fragilis & AFTOL-ID 226 & DQ986805 & DQ983487 & HQ650600 & - & - \\
\hline Sphinctrina turbinata & AFTOL-ID 1721 & EF413632 & EF413631 & - & EF413633 & - \\
\hline Spiromastix warcupii & CBS 576.63 & AB040679 & AB015768 & LN867609 & - & - \\
\hline Stachybotrys chlorohalonata & UAMH6417 & AY489712 & AY489680 & AF206273 & - & AY489607 \\
\hline Staurothele frustulenta & AFTOL-ID 697 & DQ823098 & DQ823105 & DQ826736 & DQ840560 & - \\
\hline Stegonsporium protopyriforme & CBS 117041 or D30 & EU039992 & - & NR_126119 & - & EU040017 \\
\hline Stictis confusum & Wedin 7070 & DQ401143 & - & DQ401143 & - & - \\
\hline Stictis confusum & Gilenstam 2610a & AY527327 & - & AY527327 & - & AY527306 \\
\hline Stictis radiata & Palice (ESS 21520) & AY300864 & AY300914 & - & - & - \\
\hline Sydowiella stellatifolii & CBS:119342 & EU552156 & - & EU552156 & - & - \\
\hline Sympoventuria capensis & CPC 12840 & DQ885904 & - & DQ885904 & - & - \\
\hline Tephromela atra & AFTOL-ID 780 & DQ986764 & - & HQ650606 & - & - \\
\hline Teratosphaeria associata & CBS 112224 & KF901827 & GU296200 & DQ302968 & KF902183 & KF903090 \\
\hline Thelenella antarctica & - & AY607739 & KU358979 & - & - & - \\
\hline Trapelia placodioides & KS163 & KU844623 & KU844691 & KU844758 & - & KU844395 \\
\hline Trichomerium gleosporum & MFLUCC10-0087 & JX313662 & - & JX313656 & - & - \\
\hline Tubeufia chiangmaiensis & MFLUCC110514 & KF301538 & KF301543 & KF301530 & - & KF301557 \\
\hline Umbilicaria hyperborea & Wiklund 25 & AY853399 & AY853349 & - & - & - \\
\hline
\end{tabular}


Table 1 Continued.

\begin{tabular}{|c|c|c|c|c|c|c|}
\hline \multirow{2}{*}{ Species } & \multirow{2}{*}{ Voucher/culture } & \multicolumn{5}{|c|}{ Accession numbers } \\
\hline & & LSU & SSU & ITS & $R P B 2$ & TEF1 \\
\hline Varicellaria rhodocarpa & AFTOL-ID 4890 & KJ766675 & KJ766814 & - & - & - \\
\hline Vonarxia vagans & CPC 15152 & FJ839673 & - & FJ839637 & - & - \\
\hline Xylaria acuta & AFTOL-ID 63 & AY544676 & AY544719 & DQ491493 & DQ247797 & DQ471048 \\
\hline Xylaria hypoxylon & AFTOL-ID 51 & AY544648 & AY544692 & DQ491487 & DQ470878 & DQ471042 \\
\hline Zeloasperisporium hyphopodioides & CBS 218.95 & EU035442 & - & EU035442 & - & - \\
\hline Zeloasperisporium siamemse & IFRDCC 2194 & JQ036228 & JQ036223 & - & - & - \\
\hline Zeloasperisporium wrightiae & MFLUCC 15-0225 & KT387737 & KT387738 & - & - & - \\
\hline
\end{tabular}

\section{Discussion}

Sequence data of Microthyrium-like taxa were established recently to clarify the classification of some species (Hongsanan et al. 2014a,b, 2015a,b, Ariyawansa et al. 2015). The molecular data has been used to confirm the placement of Microthyriaceae in Dothideomycetes and to establish a new family/order (Hongsanan et al. 2014a, 2015a,b, Ariyawansa et al. 2015, Hongsanan et al. 2017). Neomicrothyrium siamense was established with Microthyrium-like taxa (Fig. 3), and treated as genus incertae sedis in Microthyriales because it clustered with freshwater genus Natipusilla without similar morphological characters (Hyde et al. 2013). Hongsanan et al. (2015b) introduced two new species of the asexual genus Zeloasperisporium, which is linked to Neomicrothyrium and therefore Neomicrothyrium was synonymized under Zeloasperisporium in the new order Zeloasperisporiales (Hongsanan et al. 2015b). Chaetothyrina was placed in Micropeltidaceae based on its flattened thyriothecium, comprising interwoven hyphae, but it has 1-septate ascospores. Sequence data of two new Chaetothyrina species indicated that the genus belongs to Capnodiales as a new family, Phaeothecoidiellaceae (Hongsanan et al. 2017).

Micropeltis is the generic type of Micropeltidaceae with the type species M. applanata Mont. Wu et al. (2011) provided sequence data of M. zingiberacicola, however, it clustered separately from Microthyriaceae (represented by Microthyrium microscopicum). Therefore, Wu et al. (2011) concluded that the placement of Micropeltidaceae was uncertain, but suggested that this family probably can be placed in Microthyriales based on its morphology (Wu et al. 2011). Micropeltis zingiberacicola clustered as basal to Dothideomycetes without statistical support in previous studies (Wu et al. 2011, Hyde et al. 2013, Hongsanan et al. 2014a), however, it clustered together with Zeloasperisporiales and Natipusillales in some studies. Micropeltis dendrophthoes was established by Hongsanan et al. (2015a), and it is closely related to M. zingiberacicola in their phylogenetic tree. The placement of Micropeltidaceae in Dothideomycetes was not supported in their phylogenetic tree (Hongsanan et al. 2015a). 


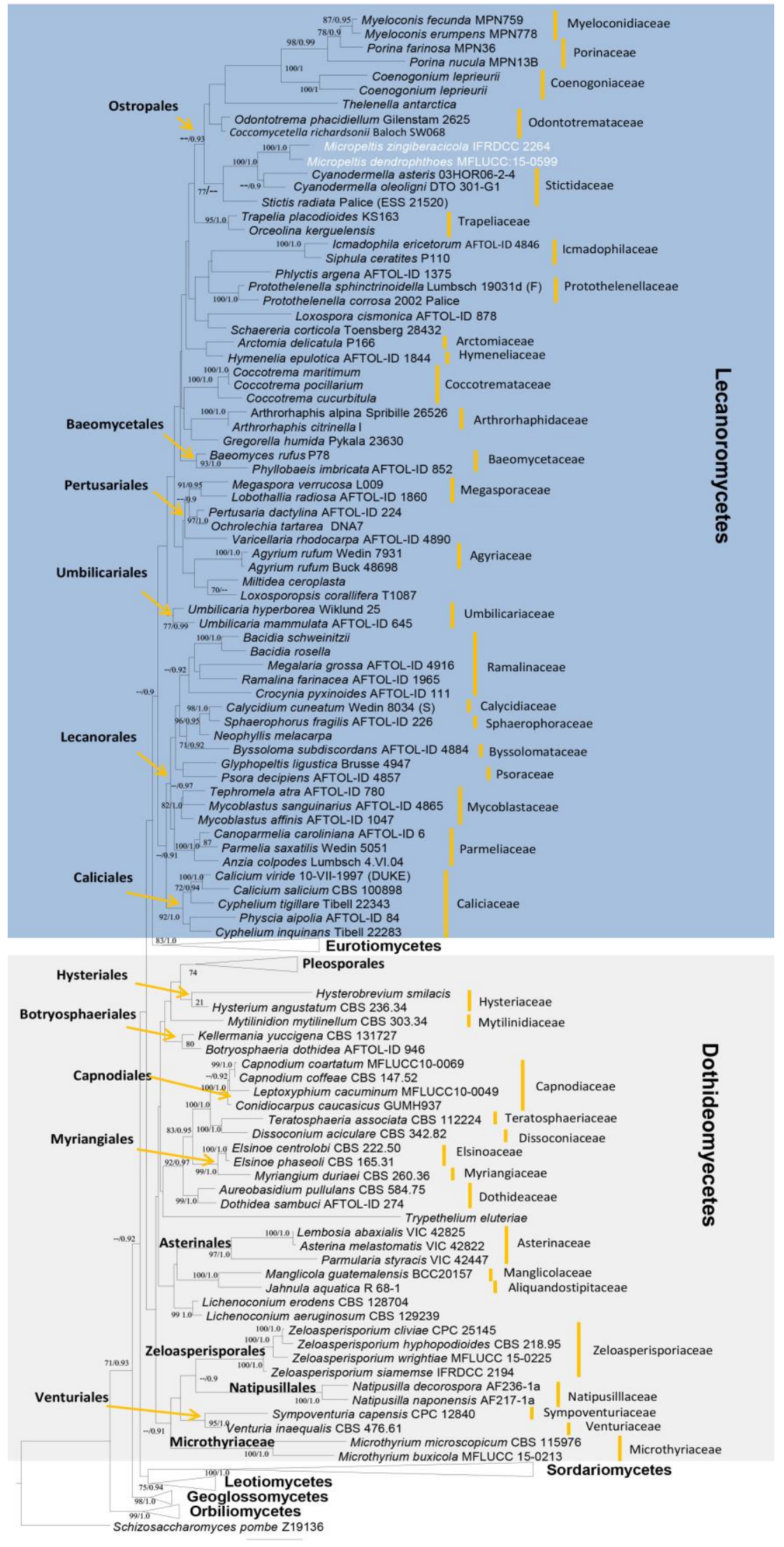

Figure 1 - RAxML maximum likelihood phylogenetic tree (LSU, RPB2, SSU and TEF1). The first set of numbers above the nodes are RAxML value expressed from 1,000 repetitions with values above $70 \%$ shown. The second set of numbers above the nodes are Bayesian posterior probabilities, with values above 0.90 shown. Strain numbers are indicated after species names. Micropeltidaceae strains are in white. 


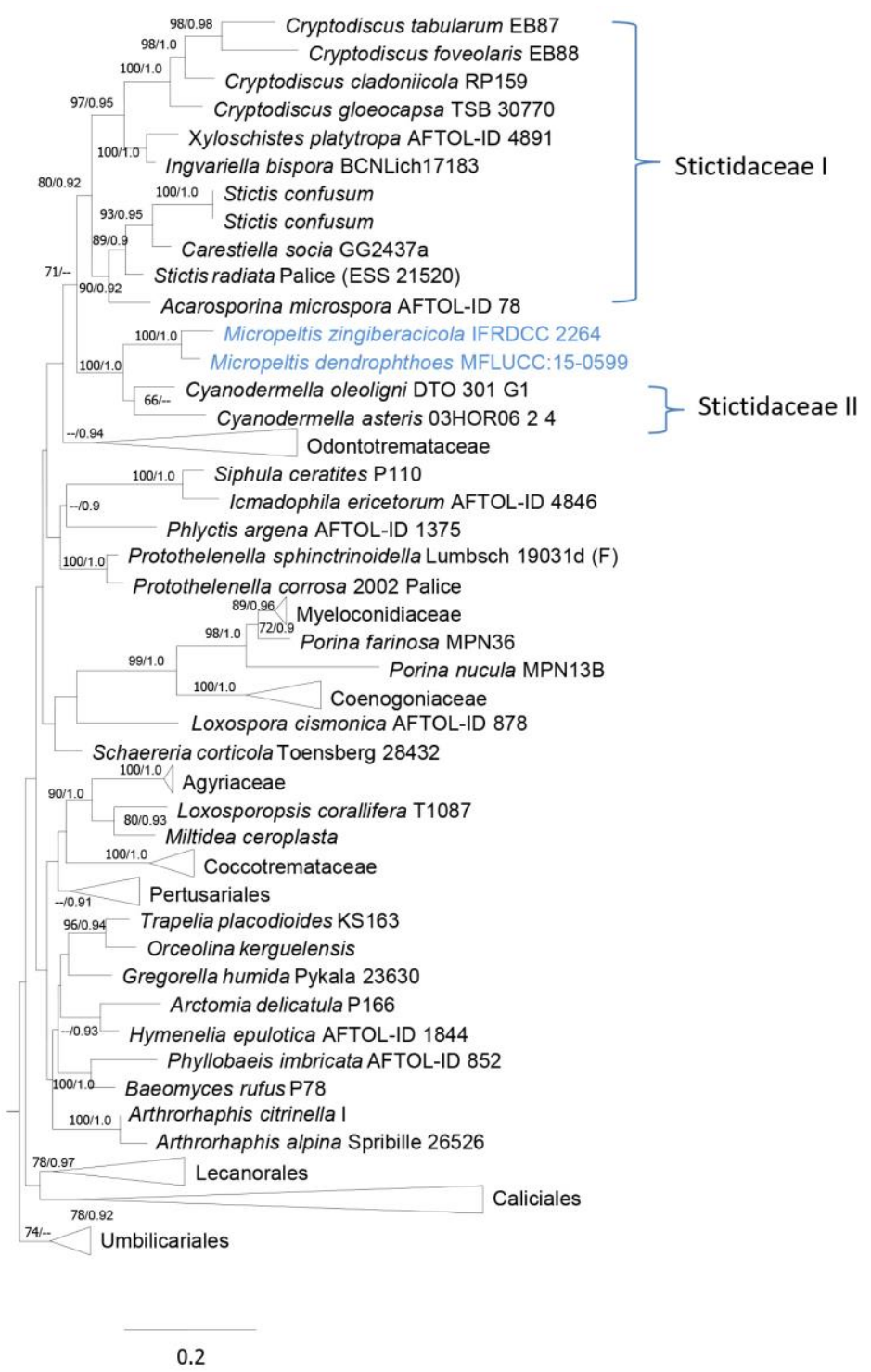

Figure 2 - RAxML maximum likelihood phylogenetic tree of Stictidaceae (ITS, LSU, SSU and TEF1). The first set of numbers above the nodes are RAxML value expressed from 1,000 repetitions with values above $70 \%$ shown. The second set of numbers above the nodes are Bayesian posterior probabilities, with values above 0.90 shown. Strain numbers are indicated after species names. Micropeltidaceae strains are in blue.

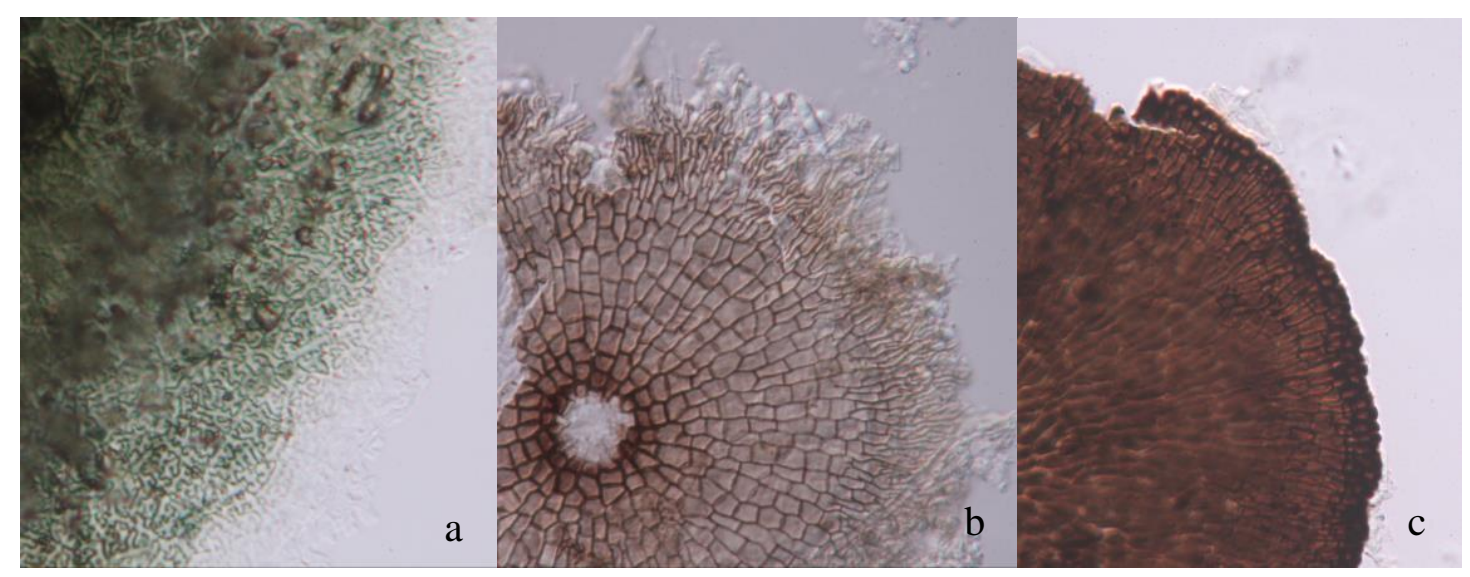

Figure 3 - a Thyriothecium of Micropeltidaceae. b Thyriothecium of Microthyriaceae. c Thyriothecium of Zeloasperisporiaceae. 
From Blast searches of both LSU and SSU gene regions in GenBank, we found that both species of Micropeltis are closely related to Cyanodermella oleoligni (DTO 301-G1) (Stictidaceae, Ostropales, Lecanoromycetes), other related species are members of Lecanoromycetes (Blast search November 2017). Phylogenetic analyses based on combined LSU, RPB2, SSU and TEF1 gene regions in this paper confirm that Microthyriaceae, represented by Microthyrium buxicola and Microthyrium microscopicum are placed in Dothideomycetes. This conforms with previous studies (Wu et al. 2011, Hyde et al. 2013, Hongsanan et al. 2014, 2015a,b). The two species from Micropeltidaceae clustered in Stictidaceae within Ostropales (Lecanoromycetes), this conforms to the same result as the Blast search. The placement of Micropeltidaceae in Dothideomycetes in previous studies was probably because they only used sequence data from Dothidiomycetes and a small number of sequences from other classes, thus, Micropeltidaceae was presented at the base of Dothidiomycetes with no statistical support. Although, phylogenetic analyses indicate that the two strains of Micropeltis species are placed with other members of Stictidaceae (Ostropales), their morphological characters are very different from other species in Ostropales. Therefore, we suggest the current placement of Micropeltidaceae as family incertae sedis in Lecanoromycetes based on its placement in phylogenetic trees and the morphological uniqueness. Further sequence data of Microthyrium-like taxa and other epiphytes (e,g Asterinaceae, Microthyriaceae, Micropeltidaceae, Phaeothecoidiellaceae, Zeloasperisporiaceae) are needed to clarify their natural classification (Wu et al. 2011, Hyde et al. 2013, Hongsanan et al. 2014b, 2015a, 2017).

We suggest that careful checking of sequence data by Blast searches is required in the future studies, not only to check the most closely related strain, but also representative strains from major classes which should be included in the first analysis to make sure the placement matches the Blast result.

\section{Acknowledgement}

Sinang Hongsanan would like to thank National Research Council of Thailand (NRCT, 2561A30702033) for supporting. Kevin D. Hyde would also like to thank NRCT for supporting research projects, Thailand's fungal diversity, solving problems and creating biotechnological products (2561A30703006).

\section{References}

Ariyawansa HA, Hyde KD, Jayasiri SC, Buyck B et al. 2015 - Fungal Diversity Notes 111-246 Taxonomic and phylogenetic contributions to fungal taxa. Fungal Diversity 75, 27-274.

Barr ME. 1987 - New taxa and combinations in the Louculoascomycetes. Mycotaxon 29, 501-505. Batista AC. 1959 - Monografia dos fungos Micropeltaceae. Publicações Instituto de Micologia da Universidade do Recife 56, 1-519.

Cai L, Guo XY, Hyde KD. 2008 - Morphological and molecular characterization of a new anamorphic genus Cheirosporium, from freshwater in China. Persoonia 20, 53-58.

Cai L, Jeewon R, Hyde KD. 2006 - Phylogenetic investigations of Sordariaceae based on multiple gene sequences and morphology. Mycological Research 110, 137-150.

Clements FE, Shear CL. 1931 - Genera of fungi 2 Vol. i-vii. H.W. Wilson, USA, New York, 496 p.

Doidge EM. 1942 - South African Microthyriaceae. Bothalia 4, 273-344.

Hall TA. 1999 - BioEdit: a user-friendly biological sequence alignment editor and analysis program for Windows 95/98/NT. Nucleic Acids Symposium Series 41, 95-98.

Hofmann TA, Piepenbring M. 2006 - New records and host plants of fly-speck fungi from Panama. Fungal Diversity 22, 55-70.

Hofmann TA. 2010 - Plant parasitic Asterinaceae and Microthyriaceae from the Neotropics (Panama). PhD thesis, The Faculty of Biological Sciences at the J.W. Goethe-University Frankfurt am in, Germany, 408 p. 
Hongsanan S, Chomnunti P, Crous PW, Chukeatirote E, Hyde KD. 2014a - Introducing Chaetothyriothecium, a new genus of Microthyriales. Phytotaxa 161, 157-164.

Hongsanan S, Li YN, Liu JK, Hofmann TA, Piepenbring M. 2014b - Revision of Asterinales. Fungal Diversity 68, 1-63.

Hongsanan S, Hyde KD, Bahkali AH, Camporesi E et al. 2015a - Fungal Biodiversity Profiles 1120. Cryptogamie Mycologie 36, 355-380.

Hongsanan S, Tian Q, Bahkali AH, Yang JB et al. 2015b-Zeloasperisporiales ord. nov., and two new species of Zeloasperisporium. Cryptogamie Mycologie 36, 301-317.

Hongsanan S, Zhao RL, Hyde KD. 2017 - A new species of Chaetothyrina on branches of mango, and introducing Phaeothecoidiellaceae fam. nov. Mycosphere 8, 137-146.

Huelsenbeck JP, Ronquist F. 2001 - MRBAYES: Bayesian inference of phylogenetic trees. Bioinformatics 17, 754-755.

Hyde KD, Jones EBG, Liu JK, Ariyawansa H et al. 2013 - Families of Dothideomycetes. Fungal Diversity 63, 1-313.

Katoh K, Asimenos G, Toh H. 2009 - Multiple alignment of DNA sequences with MAFFT. Methods in Molecular Biology 537, 39-64.

Kirk PM, Cannon PF, Minter DW, Stalpers JA. 2008 - Ainsworth \& Bisby's dictionary of the fungi, $10^{\text {th }}$ edition. CAB International, Wallingford, UK, $428 \mathrm{p}$.

Lumbsch HT, Huhndorf SM. 2010 - Outline of Ascomycota-2009. Fieldiana Life Earth Science $1,1-60$.

Luttrell ES. 1973 - Loculoascomycetes. In: Ainsworth GC, Sparrow FK, Sussman AS. (eds) The fungi. An advanced treatise. Academic Press, New York and London, 135-219 pp.

Müller E, Arx JA von. 1962 - Die Gattungen der didymosporen Pyrenomyceten. Beiträge zur Kryptogamenflora der Schweiz 11, 1-922.

Nylander JAA, Wilgenbusch JC, Warren DL, Swofford DL. 2008 - AWTY (are we there yet?): a system for graphical exploration of MCMC convergence in Bayesian phylogenetics. Bioinformatics 24: 581-583.

Rannala B, Yang Z. 1996 - Probability distribution of molecular evolutionary trees: a new method of phylogenetic inference. Journal of Molecular Evolution 43, 304-311.

Schoch CL, Crous PW, Groenewald JZ, Boehm EW et al. 2009 - A class-wide phylogenetic assessment of Dothideomycetes. Studies in Mycology 64, 1-15.

Silvestro D, Michalak I. 2012 - RaxmlGUI: a graphical front-end for RAxML. Organisms Diversity and Evolution 12, 335-337.

von Arx JA, Müller E. 1975 - A re-evaluation of the bitunicate ascomycetes with key to families and genera. Studies in Mycology 9, 1-159.

Wu X, Schoch CL, Boonmee S, Bahkali AH et al. 2011 - A reappraisal of Microthyriaceae. Fungal Diversity 51, 189-248.

Zhaxybayeva O, Gogarten JP. 2002 - Bootstrap, Bayesian probability and maximum likelihood mapping: exploring new tools for comparative genome analyses. BMC Genomics 3, 4. 